\title{
COUPLING SCHEMES FOR TWO-PARTICLE OPERATOR USED IN ATOMIC CALCULATIONS*
}

\author{
R. Juršènas and G. Merkelis \\ Institute of Theoretical Physics and Astronomy of Vilnius University, A. Goštauto 12, LT-01108 Vilnius, Lithuania \\ E-mail: jursenas@itpa.lt, merkelis@itpa.lt
}

Received 19 June 2007; revised 20 July 2007

\begin{abstract}
General formulas for a two-electron operator for open-shell atoms in a coupled tensorial form are presented. Various representations of a two-electron operator in coupled tensorial form are studied. The consideration covers the cases when the two-electron operator represents atomic interactions or some effective interaction, for instance, arising from a perturbation theory. The second quantization technique is used. General formulas are derived in $L S$ coupling. Two consistent approaches to make the coupled tensorial form for a two-particle operator are studied in detail.
\end{abstract}

Keywords: atomic spectroscopy, two-electron operator, open-shell atoms, tensorial operators

PACS: $31.15 . \mathrm{Md}, 31.15 .-\mathrm{p}, 35.15 . \mathrm{Ar}$

\section{Introduction}

For many-electron atoms and ions the ability to present a two-electron operator and its matrix elements in a form optimal for the considered task may be decisive for successful calculation solutions of many theoretical spectroscopy problems. For instance, to produce highly precise atomic structure data the largescale configuration interaction (CI) calculations [1] and many-body perturbation theory (MBPT) [2,3] are used at present. The combined approach $\mathrm{CI}+\mathrm{MBPT}$, where $\mathrm{CI}$ and MBPT methods are applied in cooperation, are intensively developed [4]. All these methods need very large sets of the matrix elements for two-electron operators associated with atomic interactions or with some effective interactions of MBPT. However, the generation of such sets is consuming much time and demands large storage space in a computer. Note that the major part of calculations is the determination of the angular parts of matrix elements (angular integration) of a two-electron operator. Therefore, a variety of techniques and methods [5] allowing to derive the formulas which enable one to write efficient (economic) computer codes of calculations of the matrix elements for a two-electron operator is developed.

Traditionally the calculations are carried out $[1,6]$ with the wave functions and operators in a coordi-

\footnotetext{
* The report presented at the 37th Lithuanian National Physics Conference, 11-13 June 2007, Vilnius, Lithuania.
}

nate representation $[7,8]$. However, recently the effective methods employing the operators given in a second quantization representation (SQR) in a coupled tensorial form have been constructively developed [9-11]. The possibility of manipulation with the creation and annihilation operators as the irreducible tensors to achieve the most favourable shape for twoelectron operator for a multishell atom gives some advantage over the coordinate representation, where the variety of forms for the tensorial products are more limited.

In SQR a coupled tensorial form of a two-particle operator was developed in a number of papers ([12-15] and references cited therein). Here we point out the paper [14] where the detailed consideration of a twoparticle operator and matrix elements has been given by suggesting the special orderings and coupling schemes for the tensorial products of creation and annihilation operators. In [15] the expressions in a coupled tensorial form for an effective two-particle operator used in a second-order MBPT were presented. However, the orderings and coupling schemes used in [15] were slightly different than in [14]. In [15] also the formula for the matrix element of a two-particle operator was given.

In the present paper we continue the previous studies [15] by considering two consistent approaches to make a coupled tensorial form for a two-particle operator. Special attention is paid to the approach when 
the expressions for the operator considered are given in terms of submatrix elements of the coupled twoelectron wave functions. This gives more freedom in choosing a convenient way for the calculations of matrix elements for open-shell atoms.

In Section 1 various ways to obtain a coupled tensorial form for a two-electron operator are studied. Sets of expressions in SQR for two-electron operator are presented. To illustrate the application of the method developed, the operator of the spin-other-orbit interaction is considered in Section 2. The Appendix contains formulas necessary to find expressions for the recoupling coefficients.

\section{Two-particle operator in coupled form}

In this section we present two different sets of expressions obtained in two approaches which use different ways of coupling for a two-particle operator given in a second quantization representation. Consider a two-particle $g_{i j}$ operator for $N$-electron atom [7]

$$
G=\sum_{i<j}^{N} g_{i j}=\frac{1}{2} \sum_{i \neq j} g_{i j}
$$

Here it is assumed that $g_{i j}=g_{j i}$. For many atomic interactions the operator $g_{12}$ is presented as the following tensorial product [7]:

$$
\begin{aligned}
g_{12} \equiv & \sum_{\substack{k_{1} \kappa_{1} \\
k_{2} \kappa_{2}}} g^{\left(k_{1} k_{2} k, \kappa_{1} \kappa_{2} \kappa\right) \Gamma} m_{\Gamma} \\
= & \sum_{\substack{k_{1} \kappa_{1} \\
k_{2} \kappa_{2}}} \sum_{m_{k} m_{\kappa}}\left[\begin{array}{ccc}
k & \kappa & \Gamma \\
m_{k} & m_{\kappa} m_{\Gamma}
\end{array}\right] g\left(r_{1}, r_{2}\right) \\
& \times\left[g_{1}^{\left(k_{1} \kappa_{1}\right)} \times g_{2}^{\left(k_{2} \kappa_{2}\right)}\right]_{m_{k} m_{\kappa}}^{(k \kappa)} \\
= & \sum_{\gamma_{1} \gamma_{2}} g\left(r_{1}, r_{2}\right)\left[g_{1}^{\left(\gamma_{1}\right)} \times g_{2}^{\left(\gamma_{2}\right)}\right]_{m_{\Gamma}}^{(\gamma) \Gamma},
\end{aligned}
$$

where $g\left(r_{1}, r_{2}\right)$ is the radial part of $g_{12}$. The first factor on the right-hand side of (2) represents the ClebschGordan coefficient. $g_{i}^{\left(k_{i} \kappa_{i}\right)}$ is an irreducible tensorial operator of ranks $k_{i}$ and $\kappa_{i}$ in $l$ and $s$ space of electron $i$, respectively. The resulting $\operatorname{rank} \Gamma=k+\kappa$. We use the following notations: $\gamma \equiv k \kappa, m_{\gamma} \equiv m_{k} m_{\kappa}$, and $\lambda \equiv l s$.

The second quantization representation of $G$ is given by the following expression [9]:

$$
\begin{aligned}
G= & \frac{1}{2} \sum_{\substack{\gamma_{1} \\
\gamma_{2} n_{i} \lambda_{i} \lambda_{j} \lambda_{j} n_{j}^{\prime} \lambda_{j}^{\prime}}} \sum_{m_{\lambda_{i} m_{i}^{\prime} m_{\lambda_{j}}} a_{\lambda_{j}^{\prime}}} a_{n_{i} \lambda_{i} m_{i}} a_{n_{j} \lambda_{j} m_{j}} a_{n_{j}^{\prime} \lambda_{j}^{\prime} m_{j}^{\prime}}^{+} a_{n_{i}^{\prime} \lambda_{i}^{\prime} m_{i}^{\prime}}^{+} \\
& \times\left\langle n_{i} \lambda_{i} m_{\lambda_{i}} n_{j} \lambda_{j} m_{\lambda_{j}}\left|g^{\left(\gamma_{1} \gamma_{2}\right)(\gamma) \Gamma}{ }_{m_{\Gamma}}\right| n_{i}^{\prime} \lambda_{i}^{\prime} m_{\lambda_{i}^{\prime}} n_{j}^{\prime} \lambda_{j}^{\prime} m_{\lambda_{j}^{\prime}}\right\rangle .
\end{aligned}
$$

Here $a_{n_{i} \lambda_{i} m_{i}}$ and $a_{n_{i}^{\prime} \lambda_{i}^{\prime} m_{i}^{\prime}}^{+}$denote electron creation and annihilation operators. In our considerations $i, j, j^{\prime}, i^{\prime}$ indicate the shells $n_{i} \lambda_{i}, n_{j} \lambda_{j}, n_{j}^{\prime} \lambda_{j}^{\prime}, n_{i}^{\prime} \lambda_{i}^{\prime}$ of $N$-electron wave fuction $\left|\left(n_{a} \lambda_{a}^{N_{a}} P_{a} n_{b} \lambda_{b}^{N_{b}} P_{b} \ldots n_{k} \lambda_{k}^{N_{k}} P_{k}\right)_{E} P M_{P}\right\rangle$ $\left(P_{k} \equiv L_{k} S_{k}, N=N_{a}+N_{b}+\ldots+N_{k}\right)$ the operator $G$ acts on. In (3), a matrix element $\left\langle n_{i} \lambda_{i} m_{\lambda_{i}} n_{j} \lambda_{j} m_{\lambda_{j}}\left|g_{m_{\Gamma}}^{\left(\gamma_{1} \gamma_{2}\right)(\gamma) \Gamma}\right| n_{i}^{\prime} \lambda_{i}^{\prime} m_{\lambda_{i}^{\prime}} n_{j}^{\prime} \lambda_{j}^{\prime} m_{\lambda_{j}^{\prime}}\right\rangle\left(\equiv\left\langle i j\left|g_{m_{\Gamma}}^{(\gamma) \Gamma}\right| i^{\prime} j^{\prime}\right\rangle\right)$ is calculated by using an uncoupled two-electron wave function $\left|n_{i} \lambda_{i} m_{i} n_{j} \lambda_{j} m_{\lambda_{j}}\right\rangle=\left|n_{i} \lambda_{i} m_{i}\right\rangle\left|n_{j} \lambda_{j} m_{\lambda_{j}}\right\rangle$.

In the present paper we develop the approaches when uncoupled (first approach) and coupled

$$
\left|n_{i} \lambda_{i} n_{j} \lambda_{j} u m_{u}\right\rangle=\sum_{m_{\lambda_{i}} m_{\lambda_{j}}}\left[\begin{array}{ccc}
\lambda_{i} & \lambda_{j} & u \\
m_{\lambda_{i}} & m_{\lambda_{j}} & m_{u}
\end{array}\right]\left|n_{i} \lambda_{i} m_{i} n_{j} \lambda_{j} m_{\lambda_{j}}\right\rangle
$$


(second approach) two-electron wave functions are used to determine the matrix element of $g_{12}^{(\gamma) \Gamma} m_{\Gamma}$ in (3). In addition, for both approaches we also study the representations of $G$ starting the summation over $m_{\lambda_{i}}$ with the antisymmetric matrix element ([16])

$$
\begin{aligned}
& \left\langle n_{i} \lambda_{i} m_{\lambda_{i}} n_{j} \lambda_{j} m_{\lambda_{j}}\left|g_{m_{\Gamma}}^{\left(k_{1} k_{2} k, \kappa_{1} \kappa_{2} \kappa\right) \Gamma}\right| n_{i}^{\prime} \lambda_{i}^{\prime} m_{\lambda_{i}^{\prime}} n_{j}^{\prime} \lambda_{j}^{\prime} m_{\lambda_{j}^{\prime}}\right\rangle_{A} \\
& =\left\langle n_{i} \lambda_{i} m_{\lambda_{i}} n_{j} \lambda_{j} m_{\lambda_{j}}\left|g_{m_{\Gamma}}^{\left(k_{1} k_{2} k, \kappa_{1} \kappa_{2} \kappa\right) \Gamma}\right| n_{i}^{\prime} \lambda_{i}^{\prime} m_{\lambda_{i}^{\prime}} n_{j}^{\prime} \lambda_{j}^{\prime} m_{\lambda_{j}^{\prime}}\right\rangle \\
& \quad-\left\langle n_{i} \lambda_{i} m_{\lambda_{i}} n_{j} \lambda_{j} m_{\lambda_{j}}\left|g_{m_{\Gamma}}^{\left(k_{1} k_{2} k, \kappa_{1} \kappa_{2} \kappa\right) \Gamma}\right| n_{j}^{\prime} \lambda_{j}^{\prime} m_{\lambda_{j}^{\prime}} n_{i}^{\prime} \lambda_{i}^{\prime} m_{\lambda_{i}^{\prime}}\right\rangle
\end{aligned}
$$

and taking into account that

$$
\begin{aligned}
G= & \frac{1}{4} \sum_{\substack{\gamma_{1} \\
\gamma_{2} n_{i}^{\prime} \lambda_{i}^{\prime} \lambda_{i}^{\prime} n_{j}^{\prime} \lambda_{j}^{\prime}}} \sum_{\substack{m_{\lambda_{i}} m_{\lambda_{j}^{\prime}} \\
m_{\lambda_{\lambda_{j}^{\prime}}} m_{\lambda_{j}}}} a_{n_{i} \lambda_{i} m_{i}} a_{n_{j} \lambda_{j} m_{j}} a_{n_{j}^{\prime} \lambda_{j}^{\prime} m_{j}^{\prime}}^{+} a_{n_{i}^{\prime} \lambda_{i}^{\prime} m_{i}^{\prime}}^{+} \\
& \times\left\langle n_{i} \lambda_{i} m_{\lambda_{i}} n_{j} \lambda_{j} m_{\lambda_{j}}\left|g^{\left(\gamma_{1} \gamma_{2}\right)(\gamma) \Gamma}\right| n_{\Gamma}^{\prime} \lambda_{i}^{\prime} m_{\lambda_{i}^{\prime}} n_{j}^{\prime} \lambda_{j}^{\prime} m_{\lambda_{j}^{\prime}}\right\rangle_{A} .
\end{aligned}
$$

Note that in the above discussion it has been assumed that the second factor in expression (3) is the matrix element of a two-electron operator $g_{12}$ with the definite tensorial structure and symmetry properties and represents some atomic interaction in an atom. However, the expressions like (3) also appear when studying the perturbation theory expansion terms (diagrams). In this case, instead of $\left(i j\left|g_{12}^{(\gamma)} \Gamma_{\Gamma}\right| i^{\prime} j^{\prime}\right)$, the sums of products of the matrix elements for operators involved in a perturbation theory expansion emerge. These products can be considered as the matrix element $\left(i j \mid\right.$ eff $\left.g_{12}^{(\gamma)} \Gamma_{\Gamma} \mid i^{\prime} j^{\prime}\right)$ of some effective operator ${ }^{\text {eff }} g_{12}^{(\gamma) m_{\Gamma}}$ with the more complicated tensorial structure and symmetry properties than those of $g_{12}^{(\gamma) \Gamma}$. Nevertheless, our consideration is developed in the way that covers the both cases.

In order to perform the sums over $m_{\lambda}$ (to present $G$ in the coupled form) one needs to take into account that $a_{m_{\lambda}}^{(\lambda)}=a_{\lambda m_{\lambda}}$ and $\widetilde{a}_{m_{\lambda}}^{(\lambda)}=(-1)^{\lambda-m_{\lambda}} a_{\lambda-m_{\lambda}}^{+}$are the irreducible tensorial operators of rank $\lambda$. After the summations are carried out, the operator $G$ can be expressed as a multiple sum of the product of two factors. One factor represents the irreducible tensorial product of creation and annihilation operators, whereas another one is a scalar (c-number) which involves the submatrix elements of $g_{i}^{\left(k_{i} \kappa_{i}\right)}$ and the recoupling coefficients arising while constructing the tensorial product.

There is a number of ways a coupled tensorial form for a two-particle operator $G$ can be obtained. The particular way and the corresponding expression for $G$ are determined by the specific coupling scheme which is used to make the irreducible tensorial product from $a_{m_{\lambda}}^{(\lambda)}$ and $\widetilde{a}_{m_{\lambda}}^{(\lambda)}$. The coupled tensorial form also depends on the tensorial structure of the second term in (3), i. e. on the matrix element $\left(i j\left|g_{12}^{(\gamma) m_{\Gamma}}\right| i^{\prime} j^{\prime}\right)$ or on the corresponding PT term which represents a particular diagram. In the present paper the creation and annihilation operators are given in the order proposed in [15]. The operators $a_{m_{\lambda}}^{(\lambda)}$ and $\widetilde{a}_{m_{\lambda}}^{(\lambda)}$ which act on the same shell are placed side by side, while the operators $a_{m_{\lambda}}^{(\lambda)}$ and $\widetilde{a}_{m_{\lambda}}^{(\lambda)}$ acting on the first (second, third) shell of many-electron wave function are situated to the left of those acting on the second (third, fourth) shell. The summation over $m_{\lambda}$ was performed by using Jucys, Levinson, and Vanagas theorems [17]. Depending on the approach developed, we have carried out several recouplings of ranks to make the irreducible tensorial product of creation and annihilation operators. In the first approach, we applied the Wigner-Eckart theorem [7] to the second term of (3) and after the summation over $m_{\gamma_{i}}$ the generalized Clebsch-Gordan coefficient was obtained. Then, the angular momenta of the generalized Clebsch-Gordan coefficient was recoupled in the way that the resulting ranks of tensorial products of $a_{m_{\lambda}}^{(\lambda)}$ and $\widetilde{a}_{m_{\lambda}}^{(\lambda)}$ associated with particular shells should be coupled in a consecutive order. More details of such technique can be found in [15]. In the second approach, firstly Eq. (4) was used to obtain the generalized Clebsch-Gordan coefficient and after that the irreducible tensorial product was build by recoupling angular momenta.

The final result of our study is a large set of the expressions for $G$. For convenience, these expressions are 
Table 1. The quantities for generation of the expressions for the operator $G$ in two-shell case.

\begin{tabular}{|c|c|c|c|c|c|}
\hline$\Delta N_{1}, \Delta N_{2}$ & $\varrho$ & $e$ & $G_{\alpha \varrho_{e}}^{\left(\Lambda_{1} \Lambda_{2}\right)(\gamma)}$ & $g g_{2 \varrho_{e}}^{b}$ & $g g_{2}^{z} \varrho_{e}$ \\
\hline 0,0 & 1 & 1 & $T 21(-1,1,-1,1)$ & $\begin{array}{l}(-1)^{\gamma_{2}+\lambda_{1}+\lambda_{2}+\Lambda_{2}} \\
\times\left(b_{1212} D+t t_{1}\right) \\
+\left(b_{1221} D_{2}^{b}+t t_{2}\right)\end{array}$ & $-c z_{1212} D_{21}^{z}$ \\
\hline$-2,2$ & 2 & 1 & $T 21(-1,-1,1,1)$ & $-b_{1122} D$ & $c z_{1122} D_{22}^{z}$ \\
\hline $2,-2$ & 5 & 1 & $T 21(1,1,-1,-1)$ & $\overline{g g_{22_{1}}^{b}}$ & $\overline{g g_{22_{1}}^{z}}$ \\
\hline \multirow[t]{2}{*}{$-1,1$} & 3 & 1 & ${ }^{x} T 22(-1,-1,1,1)$ & $\begin{array}{l}(-1)^{\lambda_{2}+\Lambda_{2}+\gamma_{1}+\gamma_{2}}\left(b_{1112}\right. \\
\left.\times \delta\left(\Lambda_{1}, \gamma_{2}\right) P+t t_{1}\right)\end{array}$ & $c z_{1112} \delta\left(\Lambda_{1}, u\right) D_{23}^{z}$ \\
\hline & & 2 & ${ }^{x} T 23(-1,-1,1,1)$ & $\begin{array}{l}(-1)^{\lambda_{1}+\Lambda_{2}+\gamma}\left(b_{1222}\right. \\
\left.\times\left.\delta\left(\Lambda_{1}, \gamma_{2}\right) P\right|_{\lambda_{1} \longleftrightarrow \lambda_{2}}+t t_{1}\right)\end{array}$ & $\begin{array}{l}c z_{1222} \delta\left(\Lambda_{1}, d\right) \\
\times\left. D_{23}^{z}\right|_{\lambda_{1} \longleftrightarrow \lambda_{2}, u \longleftrightarrow d}\end{array}$ \\
\hline \multirow[t]{2}{*}{$1,-1$} & 4 & 1 & ${ }^{x} T 24(-1,1,1,-1)$ & $(-1)^{\lambda_{1}-\Lambda_{2}} \overline{g g_{23_{1}}^{b}}$ & $(-1)^{\lambda_{1}-\Lambda_{2}} \overline{g g_{23_{1}}^{z}}$ \\
\hline & & 2 & ${ }^{x} T 25(1,-1,-1,1)$ & $(-1)^{\lambda_{2}-\Lambda_{2}} \overline{g g_{23_{2}}^{b}}$ & $(-1)^{\lambda_{2}-\Lambda_{2}} \overline{g g_{23_{2}}^{z}}$ \\
\hline
\end{tabular}

Table 2. The quantities for generation of the expressions for the operator $G$ in three-shell case.

\begin{tabular}{cllll}
\hline \hline$\Delta N_{1}, \Delta N_{2}, \Delta N_{3}$ & $\varrho$ & $G_{\alpha \varrho}^{\left(\Lambda_{1} \Lambda_{2}\right)(\gamma)}$ & $g g_{3 \varrho}^{b}$ & $g g_{3 \varrho}^{z}$ \\
\hline \hline $0,-1,1$ & 2 & $T 31(-1,1,-1,1)$ & $\begin{array}{l}-\left(b_{1213} E N+t t_{1}\right) \\
+\left(b_{1231} \delta\left(\Lambda_{1}, \gamma_{1}\right) T N+t t_{2}\right)\end{array}$ & $-\left.c z_{1213} D_{31}^{z}\right|_{u \longleftrightarrow d} \longleftrightarrow{ }^{\prime}$ \\
\hline $0,1,-1$ & 1 & $T 31(1,-1,1,-1)$ & $(-1)^{\Lambda_{1}} \overline{g g_{32}^{b}}$ & $(-1)^{\Lambda_{1}} \overline{g g_{32}^{z}}$ \\
\hline$-2,1,1$ & 4 & $T 31(-1,-1,1,1)$ & $\left(b_{1123} D_{3}^{b}+t t_{1}\right)$ & $c z_{1123} D_{33}^{z}$ \\
\hline $2,-1,-1$ & 3 & $T 31(1,1,-1,-1)$ & $\overline{g g_{34}^{b}}$ & $\overline{g g_{34}^{z}}$ \\
\hline$-1,1,0$ & 5 & $T 32(-1,1,-1,1)$ & $(-1)^{\gamma_{2}+\lambda_{2}-\lambda_{3}}\left(b_{1323} P N+t t_{1}\right)$ & $c z_{1323} D_{32}^{z}$ \\
\hline $1,-1,0$ & 6 & $T 32(1,-1,-1,1)$ & $(-1)^{\Lambda_{2}} \overline{g g_{35}^{b}}$ & $(-1)^{\Lambda_{2} \overline{g g_{35}^{z}}}$ \\
\hline$-1,-1,2$ & 7 & $T 32(-1,-1,1,1)$ & $\left(b_{1233} P N+t t_{1}\right)$ & $c z_{1233} D_{22}^{z}$ \\
\hline $1,1,-2$ & 8 & $T 32(1,1,-1,-1)$ & $\overline{g g_{37}^{b}}$ & $\overline{g g_{37}^{z}}$ \\
\hline $1,0,-1$ & 9 & $T 33(1,-1,1,-1)$ & $\left.(-1)^{\lambda_{1}+\Lambda_{1}-\Lambda_{2}} g g_{31}^{b}\right|_{\lambda_{1} \leftrightarrow \lambda_{2}}$ & $\left.(-1)^{\lambda_{1}+\Lambda_{1}-\Lambda_{2}} g g_{31}^{z}\right|_{\lambda_{1} \leftrightarrow \lambda_{2}}$ \\
\hline$-1,0,1$ & 10 & $T 33(-1,1,-1,1)$ & $\left.(-1)^{\lambda_{1}+\Lambda_{1}-\Lambda_{2}} g g_{32}^{b}\right|_{\lambda_{1} \leftrightarrow \lambda_{2}}$ & $\left.(-1)^{\lambda_{1}+\Lambda_{1}-\Lambda_{2}} g g_{32}^{z}\right|_{\lambda_{1} \leftrightarrow \lambda_{2}}$ \\
\hline$-1,2,-1$ & 11 & $T 33(-1,1,1,-1)$ & $\left.(-1)^{\lambda_{1}+\Lambda_{1}-\Lambda_{2}} g g_{33}^{b}\right|_{\lambda_{1} \leftrightarrow \lambda_{2}}$ & $\left.(-1)^{\lambda_{1}+\Lambda_{1}-\Lambda_{2}} g g_{33}^{z}\right|_{\lambda_{1} \leftrightarrow \lambda_{2}}$ \\
\hline $1,-2,1$ & 12 & $T 33(1,-1,-1,1)$ & $\left.(-1)^{\lambda_{1}+\Lambda_{1}-\Lambda_{2}} g g_{34}^{b}\right|_{\lambda_{1} \leftrightarrow \lambda_{2}}$ & $\left.(-1)^{\lambda_{1}+\Lambda_{1}-\Lambda_{2}} g g_{34}^{z}\right|_{\lambda_{1} \leftrightarrow \lambda_{2}}$ \\
\hline \hline
\end{tabular}

collected in Tables 1-3. Bellow, the detailed explanation of the notations used in tables will be given.

For both developed approaches we write

$$
\begin{aligned}
G= & \sum_{\{i\}} G_{\{i\}}+\sum_{\{i j\}} G_{\{i, j\}}+\sum_{\{i j k\}} G_{\{i, j, k\}} \\
& +\sum_{\{i j k l\}} G_{\{i, j, k, l\}} .
\end{aligned}
$$

The operators $G_{\{i\}}, G_{\{i, j\}}, G_{\{i, j, k\}}, G_{\{i, j, k, l\}}$ act in the space of the states of one, two, three, and four shells, respectively. The sums in (7) run over all possible distributions $\{i\},\{i j\},\{i j k\},\{i j k l\}$ of shells of the $N$-electron wave function. It is assumed that $i<j<k<l$. Each operator in (7) is presented as

$$
G_{\{\}}=\sum_{\substack{\Lambda_{1} \Lambda_{2} \\ \alpha \varrho_{e}}}{ }^{x} G_{\alpha \varrho_{e}}^{\left(\Lambda_{1} \Lambda_{2}\right)(\gamma) \Gamma} m_{\Gamma} g g^{x}\left(\alpha, \varrho_{e}, \Lambda_{1} \Lambda_{2}, \Gamma\right),
$$

where $x=b, z$ denotes the quantities obtained in the first and second approaches, respectively. ${ }^{x} G_{\alpha \varrho_{e}}^{\left(\Lambda_{1} \Lambda_{2}\right)(\gamma) \Gamma}{ }_{m_{\Gamma}}$ stands for the irreducible tensorial products of the 
Table 3. The quantities for generation of the expressions for the operator $G$ in four-shell case.

\begin{tabular}{|c|c|c|c|c|}
\hline$\Delta N_{1}, \Delta N_{2}, \Delta N_{3}, \Delta N_{4}$ & $\varrho$ & $G_{\alpha \varrho}^{\left(\Lambda_{1} \Lambda_{2}\right)(\gamma)}$ & $g g_{4 \varrho}^{b}$ & $g g_{4 \varrho}^{z}$ \\
\hline$-1,-1,1,1$ & 1 & $T 41(-1,-1,1,1)$ & $\begin{array}{l}-\left(b_{1243} D V+t t_{1}\right) \\
+(-1)^{\gamma_{1}+\lambda_{2}+\lambda_{3}}\left(b_{2143} D_{41}^{b}+t t_{2}\right)\end{array}$ & $-c z_{1243} D_{41}^{z}$ \\
\hline $1,1,-1,-1$ & 4 & $T 41(1,1,-1,-1)$ & $\overline{g_{41}^{b}}$ & $\overline{g_{41}^{z}}$ \\
\hline$-1,1,-1,1$ & 2 & $T 41(-1,1,-1,1)$ & $\begin{array}{l}-\left(b_{3142} D_{41}^{b}+t t_{1}\right) \\
+\left(b_{3124} \delta\left(\Lambda_{1}, \gamma_{2}\right) D_{42}^{b}+t t_{2}\right)\end{array}$ & $\begin{array}{l}(-1)^{\lambda_{1}+\lambda_{2}+\Lambda_{1}+1} \\
\times\left. c z_{3142} D_{42}^{z}\right|_{\lambda_{1} \leftrightarrow \lambda_{2}}\end{array}$ \\
\hline $1,-1,1,-1$ & 6 & $T 41(1,-1,1,-1)$ & $\overline{g_{42}^{b}}$ & $\overline{g_{42}^{z}}$ \\
\hline $1,-1,-1,1$ & 3 & $T 41(1,-1,-1,1)$ & $\begin{array}{l}(-1)^{\gamma_{1}+\lambda_{1}+\lambda_{3}} \\
\times\left(b_{3241} D V+t t_{1}\right) \\
+(-1)^{\gamma_{2}+\lambda_{1}-\lambda_{2}} \\
\times\left(b_{3214} \delta\left(\Lambda_{1}, \gamma_{2}\right) D_{42}^{b}+t t_{2}\right)\end{array}$ & $c z_{3241} D_{42}^{z}$ \\
\hline$-1,1,1,-1$ & 5 & $T 41(-1,1,1,-1)$ & $\overline{\overline{g_{43}^{b}}}$ & $\overline{g_{43}^{z}}$ \\
\hline
\end{tabular}

operators $a_{m_{\lambda}}^{(\lambda)}$ and $\widetilde{a}_{m_{\lambda}}^{(\lambda)}$ with the intermediate $\Lambda_{1}, \Lambda_{2}$ and the resulting $\gamma, \Gamma$ ranks. Further for simplicity we use the operator $G_{\alpha \varrho}^{\left(\Lambda_{1} \Lambda_{2}\right)(\gamma)} m_{\gamma}$ instead of $G_{\alpha \varrho}^{\left(\Lambda_{1} \Lambda_{2}\right)(\gamma) \Gamma} m_{\Gamma}$. Note that the submatrix element of $G_{\alpha \varrho_{e}}^{\left(\Lambda_{1} \Lambda_{2}\right)(\gamma) \Gamma}{ }_{m_{\Gamma}}$ can be easily obtained from one of $G_{\alpha \varrho}^{\left(\Lambda_{1} \Lambda_{2}\right)(\gamma)} m_{\gamma}$ by using simple relation presented e.g. in $[9,10]$. The expressions for the scalar factor $g g^{x}\left(\alpha, \varrho_{e}, \Lambda_{1} \Lambda_{2}, \Gamma\right)$ will be discussed later. The indices $\alpha$ and $\varrho_{e}$ characterize the set $\left\{n_{i} \lambda_{i} n_{j} \lambda_{j} n_{j}^{\prime} \lambda_{j}^{\prime} n_{i}^{\prime} \lambda_{i}^{\prime}\right\}$ of quantum numbers in (8). $\alpha$ specifies the number of shells the operator $G$ acts on. Note, the maximum number of shells the operator $G$ can act on is four. We collected the terms of the operator $G$ with definite $\alpha$ into groups and labelled them by $\varrho_{e}$. The operators of a particular group connect only the configuration states $\left\langle\ldots N_{1}, \ldots N_{2}, \ldots N_{3}, \ldots N_{4} \ldots\right|$ and $\left|\ldots N_{1}^{\prime}, \ldots N_{2}^{\prime}, \ldots N_{3}^{\prime}, \ldots N_{4}^{\prime} \ldots\right\rangle$ with the specific electron occupation numbers $N_{i}$ and $N_{i}^{\prime}$ of the shells the operator $G_{\alpha \varrho e}^{\left(\Lambda_{1} \Lambda_{2}\right)\left(\underset{m_{\gamma}}{\gamma}\right.}$ acts on. In Tables 1-4 the in$\operatorname{dex} \varrho$ is associated with the specific collection of numbers $\left\{\Delta N_{1}=N_{1}^{\prime}-N_{1}, \Delta N_{2}=N_{2}^{\prime}-N_{2}, \ldots\right.$, $\left.\Delta N_{4}=N_{4}^{\prime}-N_{4}\right\}$. The terms of each group $\varrho_{e}$ are characterized by the following sets of the quantum numbers:

- $s t 1=\left\{n_{i} \lambda_{i} n_{j} \lambda_{j} n_{j}^{\prime} \lambda_{j}^{\prime} n_{i}^{\prime} \lambda_{i}^{\prime}\right\}$,

- $s t 2=\left\{n_{j} \lambda_{j} n_{i} \lambda_{i} n_{i}^{\prime} \lambda_{i}^{\prime} n_{j}^{\prime} \lambda_{j}^{\prime}\right\}$,

- $s t 3=\left\{n_{i} \lambda_{i} n_{j} \lambda_{j} n_{i}^{\prime} \lambda_{i}^{\prime} n_{j}^{\prime} \lambda_{j}^{\prime}\right\}$,

- $s t 4=\left\{n_{j} \lambda_{j} n_{i} \lambda_{i} n_{j}^{\prime} \lambda_{j}^{\prime} n_{i}^{\prime} \lambda_{i}^{\prime}\right\}$.

$e$ denotes the sets with different arrays of $n_{i} \lambda_{i}, n_{j} \lambda_{j}$, $n_{j}^{\prime} \lambda_{j}^{\prime}, n_{i}^{\prime} \lambda_{i}^{\prime}$. The terms with st1 (st2) and st3 (st4) represent the direct and exchange interactions, correspondingly. Four products of creation and annihilation operators associated with the previous sets of the quantum numbers can be transformed into the single product (we denote it by $G_{\alpha \varrho_{e}}^{\left(\Lambda_{1} \Lambda_{2}\right)(\gamma) \Gamma}{ }_{m_{\Gamma}}$ ) by permuting $a_{m_{\lambda}}^{(\lambda)}$ and $\widetilde{a}_{m_{\lambda}}^{(\lambda)}$. In general, the factor $g g^{x}\left(\alpha, \varrho_{e}, \Lambda_{1} \Lambda_{2}, \Gamma\right)$ has four terms corresponding to the $s t 1$, st 2 , st 3 , st 4 sets. However, due to the symmetry properties of atomic interactions, the term in $g g^{x}\left(\alpha, \varrho_{e}, \Lambda_{1} \Lambda_{2}, \Gamma\right)$ associated with the set $s t 1$ (st3) is equal to the term desribed by st2 (st4).

The expressions for the operator $G$ and their analyses when it acts on one shell $\left(n_{i} \lambda_{i}=n_{j} \lambda_{j}=\right.$ $n_{j}^{\prime} \lambda_{j}^{\prime}=n_{i}^{\prime} \lambda_{i}^{\prime}$ ) are commonly known (see, for example, $[10,14,15])$, therefore they are not presented here.

Let us study the case when $G$ acts on two shells $(\alpha=2)$. The expressions for ${ }^{x} G_{2 \varrho_{e}}^{\left(\Lambda_{1} \Lambda_{2}\right)(\gamma) \Gamma}{ }_{m_{\Gamma}}$ and $g g^{x}\left(2, \varrho_{e}, \Lambda_{1} \Lambda_{2}, \Gamma\right)$ are presented in Table 1 . To reduce a number of expressions that should be written for $G_{\alpha \varrho_{e}}^{\left(\Lambda_{1} \Lambda_{2}\right)(\gamma)} m_{\gamma}$, we use the convention $T \alpha \beta\left(b_{1}, b_{2}, b_{3}, b_{4}\right)$ to describe the tensorial products of creation and annihilation operators with different coupling schemes. If the argument $b_{i}=-1$ or 1 , then the $i$ th operator in $T \alpha \beta$ is $a^{(\lambda)}$ or $\widetilde{a}^{(\lambda)}$. For instance, $T 21\left(b_{1}, b_{2}, b_{3}, b_{4}\right)$ describes three operators $G_{2 \varrho}^{\left(\Lambda_{1} \Lambda_{2}\right)(\gamma)} m_{\gamma}$ when $\varrho=1,2$, and 5. Note that in our study the operators ${ }^{x} G_{2 \varrho e}^{\left(\Lambda_{1} \Lambda_{2}\right)(\gamma)} m_{\gamma}$ for $x=b$ and $x=z$ are indentical for three- and fourshell cases and only for two-shell case ${ }^{x} G_{2 \varrho_{e}}^{\left(\Lambda_{1} \Lambda_{2}\right)(\gamma)} m_{\gamma}$ they are different for $x=b$ and $x=z$. Furthermore, this happens only if $\left\{\Delta N_{1}, \Delta N_{2}\right\}=\{-1,1\}$ and $\{1,-1\}$ $(\varrho=3,4)$. Exclusively for these cases there are two operators ${ }^{x} G_{2 \varrho_{e}}^{\left(\Lambda_{1} \Lambda_{2}\right)(\gamma)} m_{\gamma}(e=1,2)$ for fixed $\varrho$, and the index $e$ has been introduced to separate them. While considering ${ }^{x} G_{\alpha \varrho_{e}}^{\left(\Lambda_{1} \Lambda_{2}\right)(\gamma) \Gamma}{ }_{m_{\Gamma}}$ and $g g^{x}\left(\alpha, \varrho_{e}, \Lambda_{1} \Lambda_{2}, \Gamma\right)$ for other 
Table 4. The relations for determination of the recoupling coefficients.

\begin{tabular}{ll}
\hline Two-shell & Formula \\
\hline$D_{2}^{b}$ & $\delta\left(\Lambda_{1}, \gamma_{1}\right) \delta\left(\Lambda_{2}, \gamma_{2}\right) \Delta\left(\gamma_{1}, \gamma_{2}, \gamma\right)$ \\
\hline$D_{21}^{z}$ & $D\left(\lambda_{1}, \lambda_{2}, u, d, \gamma, \Lambda_{1}, \Lambda_{2}\right)$ \\
\hline$D_{22}^{z}$ & $\delta\left(\Lambda_{1}, u\right) \delta\left(\Lambda_{2}, d\right) \Delta(u, d, \gamma)$ \\
\hline$D_{23}^{z}$ & $(-1)^{\gamma+\lambda_{1}+\lambda_{2}+\Lambda_{1}} P\left(\lambda_{1}, \lambda_{2}, d, u, \gamma, \Lambda_{2}\right)$ \\
\hline \hline Three-shell & Formula \\
\hline$D_{3}^{b}$ & $(-1)^{\lambda_{1}+\lambda_{2}-\gamma_{2}} E N$ \\
\hline$D_{31}^{z}$ & $(-1)^{\Lambda_{1}+\gamma+u-\lambda_{1}+\lambda_{2}} E N\left(\lambda_{1}, \lambda_{2}, \lambda_{3}, u, d, \gamma, \Lambda_{1}, \Lambda_{2}\right)$ \\
\hline$D_{32}^{z}$ & $(-1)^{1+\Lambda_{2}} P N\left(\lambda_{1}, \lambda_{2}, \lambda_{3}, u, d, \gamma, \Lambda_{1}, \Lambda_{2}\right)$ \\
\hline$D_{33}^{z}$ & $T N\left(\lambda_{2}, \lambda_{3}, u, d, \gamma, \Lambda_{2}\right) \delta\left(\Lambda_{1}, u\right)$ \\
\hline \hline Four-shell & Formula \\
\hline$D_{41}^{b}$ & $(-1)^{\lambda_{1}+\lambda_{3}-\gamma_{1}+\Lambda_{1}+1} D V\left(\lambda_{2}, \lambda_{1}, \lambda_{3}, \lambda_{4}, \gamma_{1}, \gamma_{2}, \gamma, \Lambda_{1}, \Lambda_{2}\right)$ \\
\hline$D_{42}^{b}$ & $(-1)^{\lambda_{3}+\lambda_{4}-\gamma_{1}} P\left(\lambda_{3}, \lambda_{4}, \gamma_{1}, \gamma_{2}, \gamma, \Lambda_{2}\right)$ \\
\hline$D_{41}^{z}$ & $(-1)^{d+\gamma+\Lambda_{1}} \delta\left(\Lambda_{1}, u\right) P\left(\lambda_{3}, \lambda_{4}, d, u, \gamma, \Lambda_{2}\right)$ \\
\hline$D_{42}^{z}$ & $(-1)^{d+u-\lambda_{3}-\lambda_{4}+\Lambda_{1}} D V\left(\lambda_{2}, \lambda_{1}, \lambda_{3}, \lambda_{4}, u, d, \gamma, \Lambda_{1}, \Lambda_{2}\right)$ \\
\hline
\end{tabular}

cases, we skip the index $e$. The operators $G_{20_{e}}^{\left(\Lambda_{1} \Lambda_{2}\right)(\gamma)}$ are described by tensorial products with the following schemes of coupling:

$$
\begin{aligned}
& T 21(-1,1,-1,1) \\
& =\left[T^{\left(\Lambda_{1}\right)}\left(\lambda_{1}, \widetilde{\lambda}_{1}\right) \times T^{\left(\Lambda_{2}\right)}\left(\lambda_{2}, \widetilde{\lambda}_{2}\right)\right]_{m_{\gamma}}^{(\gamma)},
\end{aligned}
$$

$$
\begin{aligned}
& { }^{b} T 22(-1,-1,1,1) \\
& =\left[\left[a^{\left(\lambda_{1}\right)} \times T^{\left(\Lambda_{1}\right)}\left(\lambda_{1}, \widetilde{\lambda}_{1}\right)\right]^{\left(\Lambda_{2}\right)} \times \widetilde{a}^{\left(\lambda_{2}\right)}\right]_{m_{\gamma}}^{(\gamma)},
\end{aligned}
$$

$$
\begin{aligned}
& { }^{b} T 23(-1,-1,1,1) \\
& =\left[a^{\left(\lambda_{1}\right)} \times\left[T^{\left(\Lambda_{1}\right)}\left(\lambda_{2}, \widetilde{\lambda}_{2}\right) \times \widetilde{a}^{\left(\lambda_{2}\right)}\right]^{\left(\Lambda_{2}\right)}\right]_{m_{\gamma}}^{(\gamma)}, \\
& { }^{z} T 22(-1,-1,1,1) \\
& =\left[\left[T^{\left(\Lambda_{1}\right)}\left(\lambda_{1}, \lambda_{1}\right) \times \widetilde{a}^{\left(\lambda_{1}\right)}\right]^{\left(\Lambda_{2}\right)} \times \widetilde{a}^{\left(\lambda_{2}\right)}\right]_{m_{\gamma}}^{(\gamma)}, \\
& { }^{z} T 23(-1,-1,1,1) \\
& =\left[a^{\left(\lambda_{1}\right)} \times\left[a^{\left(\lambda_{2}\right)} \times T^{\left(\Lambda_{1}\right)}\left(\widetilde{\lambda}_{2}, \widetilde{\lambda}_{2}\right)\right]^{\left(\Lambda_{2}\right)}\right]_{m_{\gamma}}^{(\gamma)},
\end{aligned}
$$

$$
\begin{aligned}
& { }^{b} T 24(-1,1,1,-1) \\
& =\left[\left[T^{\left(\Lambda_{1}\right)}\left(\lambda_{1}, \widetilde{\lambda}_{1}\right) \times \widetilde{a}^{\left(\lambda_{1}\right)}\right]^{\left(\Lambda_{2}\right)} \times a^{\left(\lambda_{2}\right)}\right]_{m_{\gamma}}^{(\gamma)}, \\
& { }^{b} T 25(1,-1,-1,1) \\
& =\left[\widetilde{a}^{\left(\lambda_{1}\right)} \times\left[a^{\left(\lambda_{2}\right)} \times T^{\left(\Lambda_{1}\right)}\left(\lambda_{2}, \widetilde{\lambda}_{2}\right)\right]^{\left(\Lambda_{2}\right)}\right]_{m_{\gamma}}^{(\gamma)},
\end{aligned}
$$

$$
\begin{aligned}
& { }^{z} T 24(-1,1,1,-1) \\
& =\left[\left[a^{\left(\lambda_{1}\right)} \times T^{\left(\Lambda_{1}\right)}\left(\widetilde{\lambda}_{1}, \widetilde{\lambda}_{1}\right)\right]^{\left(\Lambda_{2}\right)} \times a^{\left(\lambda_{2}\right)}\right]_{m_{\gamma}}^{(\gamma)},
\end{aligned}
$$

and

$$
\begin{aligned}
& { }^{z} T 25(-1,1,1,-1) \\
& =\left[a^{\left(\lambda_{1}\right)} \times\left[T^{\left(\Lambda_{1}\right)}\left(\widetilde{\lambda}_{2}, \widetilde{\lambda}_{2}\right) \times a^{\left(\lambda_{2}\right)}\right]^{\left(\Lambda_{2}\right)}\right]_{m_{\gamma}}^{(\gamma)},
\end{aligned}
$$

where

$$
T^{(\Lambda)}\left(\lambda_{i}, \widetilde{\lambda}_{j}\right)=\left[a^{\left(\lambda_{i}\right)} \times \widetilde{a}^{\left(\lambda_{j}\right)}\right]^{(\Lambda)} .
$$


For three shells, the operators $G_{3 \varrho}^{\left(\Lambda_{1} \Lambda_{2}\right)(\gamma)} m_{\gamma}$ are defined by the tensorial products (Table 2 )

$$
\begin{aligned}
& T 31(-1,1,1,-1) \\
& =\left[\left[T^{\left(\Lambda_{1}\right)}\left(\lambda_{1}, \tilde{\lambda}_{1}\right) \times \widetilde{a}^{\left(\lambda_{2}\right)}\right]^{\left(\Lambda_{2}\right)} \times a^{\left(\lambda_{3}\right)}\right]_{m_{\gamma}}^{(\gamma)},
\end{aligned}
$$

$$
\begin{aligned}
& T 32(-1,1,-1,1) \\
& =\left[T^{\left(\Lambda_{1}\right)}\left(\lambda_{1}, \widetilde{\lambda}_{2}\right) \times T^{\left(\Lambda_{2}\right)}\left(\lambda_{3}, \widetilde{\lambda}_{3}\right)\right]_{m_{\gamma}}^{(\gamma)},
\end{aligned}
$$

and

$$
\begin{aligned}
& T 33(1,-1,1,-1) \\
& =\left[\left[\widetilde{a}^{\left(\lambda_{1}\right)} \times T^{\left(\Lambda_{1}\right)}\left(\lambda_{2}, \widetilde{\lambda}_{2}\right)\right]^{\left(\Lambda_{2}\right)} \times a^{\left(\lambda_{3}\right)}\right]_{m_{\gamma}}^{(\gamma)} .
\end{aligned}
$$

Finally, for four-shell case (Table 3)

$$
\begin{aligned}
& T 41(-1,-1,1,1) \\
& =\left[\left[T^{\left(\Lambda_{1}\right)}\left(\lambda_{1}, \lambda_{2}\right) \times \widetilde{a}^{\left(\lambda_{3}\right)}\right]^{\left(\Lambda_{2}\right)} \times \widetilde{a}^{\left(\lambda_{4}\right)}\right]_{m_{\gamma}}^{(\gamma)} .
\end{aligned}
$$

Now consider the factor $g g^{x}\left(\alpha, \varrho, \Lambda_{1}, \Lambda_{2}, \gamma\right)$. In the first approach we obtained that

$$
\begin{aligned}
g g^{b} \equiv & g g^{b}\left(\alpha, \varrho, \Lambda_{1}, \Lambda_{2}, \gamma\right) \\
= & \sum_{\gamma_{1} \gamma_{2}}\left\{\left[M D_{\alpha \varrho}\left(\gamma, \lambda_{i}, \lambda_{j}, \lambda_{j}^{\prime}, \lambda_{i}^{\prime}, \gamma_{1}, \gamma_{2}, \Lambda_{1}, \Lambda_{2}\right)\right.\right. \\
& \left.\times b\left(\gamma, \lambda_{i}, \lambda_{j}, \lambda_{j}^{\prime}, \lambda_{i}^{\prime}, \gamma_{1}, \gamma_{2}\right)+t t_{1}\right] \\
& +\left[M E_{\alpha \varrho}\left(\gamma, \lambda_{i}, \lambda_{j}, \lambda_{j}^{\prime}, \lambda_{i}^{\prime}, \gamma_{1}, \gamma_{2}, \Lambda_{1}, \Lambda_{2}\right)\right. \\
& \left.\left.\times b\left(\gamma, \lambda_{i}, \lambda_{j}, \lambda_{i}^{\prime}, \lambda_{j}^{\prime}, \gamma_{1}, \gamma_{2}\right)+t t_{2}\right]\right\} .
\end{aligned}
$$

Here

$$
\begin{aligned}
b_{i j j^{\prime} i^{\prime}} \equiv & b_{\lambda i \lambda j \lambda^{\prime} j \lambda^{\prime} i}=b\left(\gamma, \lambda_{i}, \lambda_{j}, \lambda_{j}^{\prime}, \lambda_{i}^{\prime}, \gamma_{1}, \gamma_{2}\right) \\
= & \left(n_{i} l_{i} n_{j} l_{j}\left|g\left(r_{1}, r_{2}\right)\right| n_{i}^{\prime} l_{i}^{\prime} n_{j}^{\prime} l_{j}^{\prime}\right) \\
& \times \frac{1}{2} \frac{\left[\lambda_{i}, \lambda_{j}\right]^{1 / 2}}{\left[\gamma_{1}, \gamma_{2}\right]^{1 / 2}} \Delta\left(\gamma, \gamma_{1}, \gamma_{2}\right)\left[\lambda_{i}\left\|g^{\left(\gamma_{1}\right)}\right\| \lambda_{i}^{\prime}\right] \\
& \times\left[\lambda_{j}\left\|g^{\left(\gamma_{2}\right)}\right\| \lambda_{j}^{\prime}\right],
\end{aligned}
$$

where $\left(n_{i} l_{i} n_{j} l_{j}\left|g\left(r_{1}, r_{2}\right)\right| n_{i}^{\prime} l_{i}^{\prime} n_{j}^{\prime} l_{j}^{\prime}\right)$ is a radial part of $G . \Delta\left(\gamma, \gamma_{1}, \gamma_{2}\right)$ denotes a triangular delta and $\left[\lambda_{i}\left\|g^{(\gamma)}\right\| \lambda_{j}\right]$ is the submatrix element of $g^{(\gamma)}[7]$. A shorthand notation $[k] \equiv 2 k+1$ is used wherever representation dimensions appear. The expressions for $g g^{b}\left(\alpha, \varrho, \Lambda_{1}, \Lambda_{2}, \gamma\right)$ are presented in the fifth column of Table 1 and the fourth column of Tables 2 and 3. The factors $M D_{\alpha \varrho}$ and $M E_{\alpha \varrho}$ arrive due to the recoupling procedures. The explicit expressions for these factors are found by using the relations of Table 4 and the expressions (A1)-(A6) of the Appendix. The formula for the term $t t_{1}\left(t t_{2}\right)$ linked to $s t 2(s t 4)$ is obtained from the expression of the first term in braces associated with $s t 1$ (st3) by replacing $b_{i j j^{\prime} i^{\prime}}$ $\left(b_{i j i^{\prime} j^{\prime}}\right)$ with $b_{j i i^{\prime} j^{\prime}}\left(b_{j i j^{\prime} i^{\prime}}\right)$, interchanging $\gamma_{1}$ and $\gamma_{2}$ $\left(\gamma_{1} \leftrightarrow \gamma_{2}\right)$, and multiplying the obtained formula by the factor $(-1)^{\gamma_{1}+\gamma_{2}-\gamma}=\omega$. In addition, the notation $\overline{g g_{\alpha \varrho^{\prime}}^{b}}$ in tables means that the expression for $g g_{\alpha \varrho}^{b}$ is found from the one of $g g_{\alpha \varrho^{\prime}}^{b}$ when $b_{i j j^{\prime} i^{\prime}}$ is replaced with $b_{i^{\prime} j^{\prime} j i}$ and the obtained formula is multiplied by $(-1)^{\lambda_{i}+\lambda_{j}+\lambda_{i}^{\prime}+\lambda_{j}^{\prime}-\gamma_{1}-\gamma_{2}}$. Note again that for atomic interactions where $g_{i j}=g_{j i}$, the term $t t_{1}\left(t t_{2}\right)$ is equal to the term associated with $s t 1$ (st3). The expression for $G$ for effective interaction eff $g_{12}^{(\gamma)} \Gamma_{\Gamma}$ is obtained when the factor $b\left(\gamma, \lambda_{i}, \lambda_{j}, \lambda_{j}^{\prime}, \lambda_{i}^{\prime}, \gamma_{1}, \gamma_{2}\right)$ is replaced with the submatrix element of $\left(\left.i j\right|^{\text {eff }} g_{12}^{(\gamma) \Gamma} \mid i_{\Gamma}^{\prime} j^{\prime}\right)$. To obtain the expression for $g g^{b}\left(\alpha, \varrho, \Lambda_{1} \Lambda_{2}, \gamma\right)$ in the case of the antisymmetric matrix element (5), the factor $b\left(\gamma, \lambda_{i}, \lambda_{j}, \lambda_{j}^{\prime}, \lambda_{i}^{\prime}, \gamma_{1}, \gamma_{2}\right)$ of the term with $s t 1$ in (23) must be replaced with the

$$
\begin{aligned}
b_{i j j^{\prime} i^{\prime}}^{A}= & \frac{1}{2}\left[b\left(\gamma, \lambda_{i}, \lambda_{j}, \lambda_{j}^{\prime}, \lambda_{i}^{\prime}, \gamma_{1}, \gamma_{2}\right)\right. \\
& -\sum_{p_{1} p_{2}}(-1)^{\lambda_{i}^{\prime}-\lambda_{j}^{\prime}-\gamma_{2}-p_{2}}\left[\gamma_{1}, \gamma_{2}, p_{1}, p_{2}\right]^{1 / 2} \\
& \left.\times\left\{\begin{array}{c}
\lambda_{i} \lambda_{j}^{\prime} p_{1} \\
\lambda_{i}^{\prime} \lambda_{j} p_{2} \\
\gamma_{1} \gamma_{2}
\end{array}\right\} b\left(\gamma, \lambda_{i}, \lambda_{j}, \lambda_{i}^{\prime}, \lambda_{j}^{\prime}, p_{1}, p_{2}\right)\right],
\end{aligned}
$$

whereas the remaining terms in (23) (corresponding to $s t 2$, st3, and $s t 4)$ must be skipped out.

To illustrate the derivation of a particular expression for $G$, consider a two-shell case $(\alpha=2)$ with the electron occupation numbers $N_{1}, N_{2}$ and $N_{1}^{\prime}=N_{1}-$ $1, N_{2}^{\prime}=N_{2}+1(\varrho=3),\left\{\Delta N_{1}, \Delta N_{2}\right\}=\{-1,1\}$. By using Eq. (8) and the formulas in the rows of Table 1 
corresponding to $\varrho=3$, and by recovering the notations introduced above, we find that

$$
\begin{aligned}
& G_{\{1,2\}} \\
& =\sum_{\Lambda_{1} \Lambda_{2} \gamma_{1} \gamma_{2}}\left\{{ }^{b} T 22(-1,-1,1,1)(-1)^{\lambda_{2}+\Lambda_{2}+\gamma_{1}+\gamma_{2}}\right. \\
& \quad \times\left[b_{1112} \delta\left(\Lambda_{1}, \gamma_{2}\right) P+\left.b_{1121} \omega \delta\left(\Lambda_{1}, \gamma_{1}\right) P\right|_{\gamma_{1} \leftrightarrow \gamma_{2}}\right] \\
& \quad+{ }^{b} T 23(-1,-1,1,1)(-1)^{\lambda_{1}+\Lambda_{2}+\gamma} \\
& \quad \times\left[\left.b_{1222} \delta\left(\Lambda_{1}, \gamma_{2}\right) P\right|_{\lambda_{1} \leftrightarrow \lambda_{2}}\right. \\
& \left.\left.\quad+\left.b_{2122} \omega \delta\left(\Lambda_{1}, \gamma_{1}\right) P\right|_{\gamma_{1} \leftrightarrow \gamma_{2}, \lambda_{1} \leftrightarrow \lambda_{2}}\right]\right\} .
\end{aligned}
$$

Let us consider the second approach, when at the beginning of the construction of a coupled form of $G$ the coupled two-electron wave functions (4) are used. As it was noted earlier, except for the two-shell case, the tensorial products ${ }^{z} G_{\alpha \varrho e}^{\left(\Lambda_{1} \Lambda_{2}\right)(\gamma)} m_{\gamma}$ are equal to ${ }^{b} G_{\alpha \varrho e}^{\left(\Lambda_{1} \Lambda_{2}\right) \underset{m_{\gamma}}{(\gamma)}}$. The general formula for $g g^{x}(x=z)$ is presented as follows:

$$
\begin{aligned}
g g^{z} \equiv & g g^{z}\left(\alpha, \varrho, \Lambda_{1} \Lambda_{2}, \gamma\right) \\
= & \sum_{u d} U E_{\alpha \varrho}\left(\Lambda_{1}, \Lambda_{2}, \gamma, \lambda_{i}, \lambda_{j}, \lambda_{j}^{\prime}, \lambda_{i}^{\prime}, u, d\right) \\
& \quad \times c z\left(\gamma, \lambda_{i}, \lambda_{j}, \lambda_{j}^{\prime}, \lambda_{i}^{\prime}, u, d\right) .
\end{aligned}
$$

The expressions of factors $U E_{\alpha \varrho}\left(\gamma, \lambda_{i}, \lambda_{j}, \lambda_{j}^{\prime}, \lambda_{i}^{\prime}, u, d\right)$, which represent the recoupling coefficients in the second approach, are given in the last column of Tables $1-3$. The factor

$$
\begin{aligned}
& c z_{i j j^{\prime} i^{\prime}} \equiv c z\left(\gamma, \lambda_{i}, \lambda_{j}, \lambda_{j}^{\prime}, \lambda_{i}^{\prime}, u, d\right) \\
& =z_{i j j^{\prime} i^{\prime}}+[1-\delta(i, j)](-1)^{\lambda_{i}+\lambda_{j}-u+1} z_{j i j^{\prime} i^{\prime}} \\
& +\left[1-\delta\left(i^{\prime}, j^{\prime}\right)\right](-1)^{\lambda_{i}^{\prime}+\lambda_{j}^{\prime}-d+1} z_{i j i^{\prime} j^{\prime}}+[1-\delta(i, j)] \\
& \times\left[1-\delta\left(i^{\prime}, j^{\prime}\right)\right](-1)^{\lambda_{i}+\lambda_{j}-u+\lambda_{i}^{\prime}+\lambda_{j}^{\prime}-d} z_{j i i^{\prime} j^{\prime}}
\end{aligned}
$$

In the case of (2),

$$
\begin{aligned}
& z_{i j j^{\prime} i^{\prime}} \equiv z\left(\gamma, \lambda_{i}, \lambda_{j}, \lambda_{j}^{\prime}, \lambda_{i}^{\prime}, u, d\right) \\
& =\frac{1}{2}(-1)^{\lambda_{i}^{\prime}+\lambda_{j}^{\prime}-d} \frac{[u]^{1 / 2}}{[\gamma]^{1 / 2}} \\
& \times \sum_{\gamma_{1} \gamma_{2}}\left[n_{i} \lambda_{i} n_{j} \lambda_{j} u\left\|\left[g^{\left(\gamma_{1}\right)} \times g^{\left(\gamma_{2}\right)}\right]^{(\gamma)}\right\| n_{i}^{\prime} \lambda_{i}^{\prime} n_{j}^{\prime} \lambda_{j}^{\prime} d\right] \\
& \times\left(n_{i} l_{i} n_{j} l_{j}\left|g\left(r_{1}, r_{2}\right)\right| n_{i}^{\prime} l_{i}^{\prime} n_{j}^{\prime} l_{j}^{\prime}\right) .
\end{aligned}
$$

A submatrix element of operator $\left[g^{\left(\gamma_{1}\right)} \times g^{\left(\gamma_{2}\right)}\right]^{(\gamma)}$ equals to [17]

$$
\begin{aligned}
& {\left[\lambda_{i} \lambda_{j} u\left\|\left[g^{\left(\gamma_{1}\right)} \times g^{\left(\gamma_{2}\right)}\right]^{(\gamma)}\right\| \lambda_{i}^{\prime} \lambda_{j}^{\prime} d\right]=\left[\lambda_{i}, \lambda_{j}, \gamma, d\right]^{1 / 2}} \\
& \times\left\{\begin{array}{c}
\lambda_{i}^{\prime} \lambda_{j}^{\prime} d \\
\gamma_{1} \gamma_{2} \gamma \\
\lambda_{i} \lambda_{j} u
\end{array}\right\}\left[\lambda_{i}\left\|g^{\left(\gamma_{1}\right)}\right\| \lambda_{i}^{\prime}\right]\left[\lambda_{j}\left\|g^{\left(\gamma_{2}\right)}\right\| \lambda_{j}^{\prime}\right] \cdot(30)
\end{aligned}
$$

For atomic interactions the first (second) term in (28) gives the same contribution as the fourth (third) term. When studying the effective interaction,

$$
\begin{aligned}
& z\left(\gamma, \lambda_{i}, \lambda_{j}, \lambda_{j}^{\prime}, \lambda_{i}^{\prime}, u, d\right)=(-1)^{\lambda_{i}^{\prime}+\lambda_{j}^{\prime}-d} \frac{[u]^{1 / 2}}{[\gamma]^{1 / 2}} \\
& \times\left[\lambda_{i} \lambda_{j} u\left\|^{\mathrm{eff}} g_{12}^{(\gamma) \Gamma m_{\Gamma}}\right\| \lambda_{j}^{\prime} \lambda_{i}^{\prime} d\right] .
\end{aligned}
$$

For the antisymmetric coupled two-electron wave functions the submatrix element of the operator $g_{m_{\gamma}}^{(\gamma)}$ is given by

$$
\begin{aligned}
& {\left[\lambda_{i} \lambda_{j} u\left\|g^{(\gamma)}\right\| \lambda_{i}^{\prime} \lambda_{j}^{\prime} d\right]_{A}=} \\
& \sum_{\gamma_{1} \gamma_{2}}\left(n_{i} l_{i} n_{j} l_{j}\left|g\left(r_{1}, r_{2}\right)\right| n_{i}^{\prime} l_{i}^{\prime} n_{j}^{\prime} l_{j}^{\prime}\right) \\
& \times\left[\lambda_{i} \lambda_{j} u\left\|\left[g^{\left(\gamma_{1}\right)} \times g^{\left(\gamma_{2}\right)}\right]^{(\gamma)}\right\| \lambda_{i}^{\prime} \lambda_{j}^{\prime} d\right] \\
& -(-1)^{\lambda_{i}^{\prime}+\lambda_{j}^{\prime}-\gamma} \sum_{\gamma_{1} \gamma_{2}}\left(n_{i} l_{i} n_{j} l_{j}\left|g\left(r_{1}, r_{2}\right)\right| n_{j}^{\prime} l_{j}^{\prime} n_{i}^{\prime} l_{i}^{\prime}\right) \\
& \times\left[\lambda_{i} \lambda_{j} u\left\|\left[g^{\left(\gamma_{1}\right)} \times g^{\left(\gamma_{2}\right)}\right]^{(\gamma)}\right\| \lambda_{j}^{\prime} \lambda_{i}^{\prime} d\right] .
\end{aligned}
$$


Then

$$
\begin{aligned}
& c z\left(\gamma, \lambda_{i}, \lambda_{j}, \lambda_{j}^{\prime}, \lambda_{i}^{\prime}, u, d\right)= \\
& \frac{1}{4}(-1)^{\lambda_{i}^{\prime}+\lambda_{j}^{\prime}-d} \frac{[u]^{1 / 2}}{[\gamma]^{1 / 2}}\left[\lambda_{i} \lambda_{j} u\left\|g^{(\gamma)}\right\| \lambda_{i}^{\prime} \lambda_{j}^{\prime} d\right]_{A} .
\end{aligned}
$$

In tables the notation $\overline{g g_{\alpha \varrho^{\prime}}^{z}}$ means that the expression for $g g_{\alpha \varrho}^{z}$ is found from the one for $\left.g g_{\alpha \varrho^{\prime}}^{z}\right|_{u \leftrightarrow d}$, where $z_{i j j^{\prime} i^{\prime}}$ is replaced with $z_{i^{\prime} j^{\prime} j i}$ and the obtained formula is multiplied by the phase factor $(-1)^{\lambda_{i}+\lambda_{j}+\lambda_{i}^{\prime}+\lambda_{j}^{\prime}-\gamma}$.

Consider the special case of $G$ when $\alpha=2, \varrho=3$. By using the similar steps as in the first approach, we can write

$$
\begin{aligned}
& G_{\{1,2\}} \\
& =\sum_{\Lambda_{1} \Lambda_{2} u d}\left[{ }^{z} T 22(-1,-1,1,1)\right. \\
& \quad \times c z\left(\gamma, \lambda_{1}, \lambda_{1}, \lambda_{1}, \lambda_{2}, u, d\right) \delta\left(\Lambda_{1}, u\right) D_{23}^{z} \\
& \quad+{ }^{z} T 23(-1,-1,1,1) c z\left(\gamma, \lambda_{1}, \lambda_{2}, \lambda_{2}, \lambda_{2}, u, d\right) \\
& \left.\quad \times\left.\delta\left(\Lambda_{1}, d\right) D_{23}^{z}\right|_{\lambda_{1} \leftrightarrow \lambda_{2}, u \leftrightarrow d}\right] .
\end{aligned}
$$

In summary, the expression for a specific term of the operator $G$ is obtained in steps. In the first step, the shells the operator $G$ acts on (i. e. those defined by $\alpha$ and $\varrho_{e}$ in (8)) are selected by considering

$$
\left\langle\ldots N_{1}, \ldots N_{2}, \ldots N_{3}, \ldots N_{4} \ldots\right|
$$

and

$$
\left|\ldots N_{1}^{\prime}, \ldots N_{2}^{\prime}, \ldots N_{3}^{\prime}, \ldots N_{4}^{\prime} \ldots\right\rangle
$$

which are used to calculate the matrix element. After that, the expressions for ${ }^{x} G_{\alpha \varrho_{e}}^{\left(\Lambda_{1} \Lambda_{2}\right)(\gamma) \Gamma}{ }_{m_{\Gamma}}$ and $g g^{x}\left(\alpha, \varrho_{e}, \Lambda_{1} \Lambda_{2}, \Gamma\right)$ are chosen from Tables $1-3$. In the second step, the final formulae for $g g^{x}\left(\alpha, \varrho_{e}, \Lambda_{1} \Lambda_{2}, \Gamma\right)$ are obtained from Table 4 and the expressions $(A 1)-$ (A6) given in Appendix. In the case of the consecutive coupling of the resulting moments of the shells of many-electron wave function, the formulae for matrix elements of operators $G_{\alpha \varrho}^{\left(\Lambda_{1} \Lambda_{2}\right) \underset{m_{\gamma}}{(\gamma)}}$ can be easily obtained by using the general formula presented in [15].

\section{Spin-other-orbit interaction}

To illustrate the application of the second approach, consider the operator of the spin-other-orbit interaction $(\gamma \equiv k \kappa=11$ and $\Gamma=0)[7]$ :

$$
\begin{aligned}
g_{12}= & H_{12}^{\text {soo }} \\
= & \frac{\mathrm{i} \alpha^{2}}{\sqrt{2}} \sum_{k K K^{\prime}}[k]^{-1 / 2}\left(K^{\prime}+k+1\right)^{-1 / 2}\left(1+P_{12}\right) \\
\times & {\left[\left(\left[P_{1}^{\left(K 1 K^{\prime}\right)} \times C_{2}^{(k)}\right]^{(1)} \cdot\left(s_{1}^{(1)}+2 s_{2}^{(1)}\right)\right)\right.} \\
& \times \frac{1}{r_{1}}\left(D_{1}^{K} \frac{r_{<}^{k}}{r_{>}^{k+1}}\right) \\
+ & \left(\left[C_{1}^{\left(K 1 K^{\prime}\right)} \times C_{2}^{(k)}\right]^{(1)} \cdot\left(s_{1}^{(1)}+2 s_{2}^{(1)}\right)\right) \\
& \left.\times\left(D_{1}^{K} \frac{r_{<}^{k}}{r_{>}^{k+1}}\right) \frac{\partial}{\partial r_{1}}\right]
\end{aligned}
$$

where

$$
D^{K}=\frac{\partial}{\partial r}+\left\{\begin{array}{c}
\frac{k+1}{r}, K=k-1 \\
-\frac{k}{r}, \quad K=k+1
\end{array}\right.
$$

and

$$
\left\{\begin{aligned}
P^{\left(K 1 K^{\prime}\right)} & =\mathrm{i} \sqrt{2} a_{K K^{\prime}}\left[C^{(K)} \times\left[C^{(1)} \times L^{(1)}\right]^{(1)}\right]^{\left(K^{\prime}\right)}, \\
C^{\left(K 1 K^{\prime}\right)} & =a_{K K^{\prime}}\left[C^{(K)} \times C^{(1)}\right]^{\left(K^{\prime}\right)}, \\
a_{K K^{\prime}}= & \mathrm{i}^{k+1-K}\left[k, K, K^{\prime}\right]^{1 / 2} \sqrt{K^{\prime}+k+1} \\
& \times\left[\begin{array}{ccc}
k & 1 & K \\
0 & 0 & 0
\end{array}\right]\left\{\begin{array}{ccc}
k & 1 & K \\
1 & K^{\prime} & 1
\end{array}\right\} .
\end{aligned}\right.
$$

Note that $K, K^{\prime}=k+1, k+1 ; k+1, k ; k-1, k ; k-$ $1, k-1$. Keeping in mind (29) $\left(u \equiv L S\right.$ and $\left.d \equiv L^{\prime} S^{\prime}\right)$ and (35), we write that

$$
\begin{aligned}
& z_{i j j^{\prime} i^{\prime}}^{\text {soo }} \\
& =\left[1+2(-1)^{S+S^{\prime}}\right]\left[1+(-1)^{S+S^{\prime}} P\left(k_{1} \longleftrightarrow k_{2}\right)\right] \\
& \quad \times\left(z_{i j j^{\prime} i^{\prime}}^{I}+z_{i j j^{\prime} i^{\prime}}^{I I I}\right) .
\end{aligned}
$$


The factors

$$
\begin{aligned}
& z_{i j j^{\prime} i^{\prime}}^{I}=-\sqrt{3} \sum_{\substack{k K K^{\prime} \\
k_{1}=K^{\prime} \\
k_{2}=k}}[k]^{-1 / 2}\left(K^{\prime}+k+1\right)^{-1 / 2} \\
& \times(-1)^{l_{i^{\prime}}+l_{j^{\prime}}+L^{\prime}+1}\left[l_{i}, l_{j}, L, S, L^{\prime}, S^{\prime}\right]^{1 / 2} \\
& \times I_{K}\left(i i^{\prime}, j j^{\prime}\right)\left[l_{i}\left\|P^{\left(K 1 k_{1}\right)}\right\| l_{i^{\prime}}\right]\left[l_{j}\left\|C^{\left(k_{2}\right)}\right\| l_{j^{\prime}}\right] \\
& \times\left\{\begin{array}{ccc}
1 / 2 & 1 / 2 & 1 \\
S & S^{\prime} & 1 / 2
\end{array}\right\}\left\{\begin{array}{ccc}
l_{i^{\prime}} & l_{j^{\prime}} & L^{\prime} \\
k_{1} & k_{2} & 1 \\
l_{i} & l_{j} & L
\end{array}\right\}
\end{aligned}
$$

and

$$
\begin{aligned}
z_{i j j^{\prime} i^{\prime}}^{I I I}= & -\sqrt{3} \sum_{\substack{k K K^{\prime} \\
k_{1}=K^{\prime} \\
k_{2}=k}}[k]^{-1 / 2}\left(K^{\prime}+k+1\right)^{-1 / 2} \\
& \times(-1)^{l_{i^{\prime}}+l_{j^{\prime}}+L^{\prime}+1}\left[l_{i}, l_{j}, L, S, L^{\prime}, S^{\prime}\right]^{1 / 2} \\
& \times I_{K}^{\prime}\left(i i^{\prime}, j j^{\prime}\right)\left[l_{i}\left\|C^{\left(K 1 k_{1}\right)}\right\| l_{i^{\prime}}\right]\left[l_{j}\left\|C^{\left(k_{2}\right)}\right\| l_{j^{\prime}}\right] \\
& \times\left\{\begin{array}{ccc}
1 / 2 & 1 / 2 & 1 \\
S & S^{\prime} & 1 / 2
\end{array}\right\}\left\{\begin{array}{lll}
l_{i^{\prime}} & l_{j^{\prime}} L^{\prime} \\
k_{1} & k_{2} & 1 \\
l_{i} & l_{j} & L
\end{array}\right\}
\end{aligned}
$$

describe $z_{i j j^{\prime} i^{\prime}}$ in case of the coupled two-electron wave functions. The radial integrals $I_{K}\left(i i^{\prime}, j j^{\prime}\right)$ and $I_{K}^{\prime}\left(i i^{\prime}, j j^{\prime}\right)[7]$ are equal to

$$
\begin{aligned}
& I_{K}\left(i i^{\prime}, j j^{\prime}\right) \equiv I_{K}\left(n_{i} l_{i} n_{i^{\prime}} l_{i^{\prime}}, n_{j} l_{j} n_{j^{\prime}} l_{j^{\prime}}\right) \\
& =\frac{\alpha^{2}}{4}\left(n_{i} l_{i} n_{j} l_{j}\left|\frac{1}{r_{1}}\left(D_{1}^{K} \frac{r_{<}^{k}}{r_{>}^{k+1}}\right)\right| n_{i^{\prime}} l_{i^{\prime}} n_{j^{\prime}} l_{j^{\prime}}\right)
\end{aligned}
$$

and

$$
\begin{aligned}
& I_{K}^{\prime}\left(i i^{\prime}, j j^{\prime}\right) \equiv I_{K}^{\prime}\left(n_{i} l_{i} n_{i^{\prime}} l_{i^{\prime}}, n_{j} l_{j} n_{j^{\prime}} l_{j^{\prime}}\right) \\
& =\frac{\alpha^{2}}{4}\left(n_{i} l_{i} n_{j} l_{j}\left|\left(D_{1}^{K} \frac{r_{<}^{k}}{r_{>}^{k+1}}\right) \frac{\partial}{\partial r_{1}}\right| n_{i^{\prime}} l_{i^{\prime}} n_{j^{\prime}} l_{j^{\prime}}\right),
\end{aligned}
$$

respectively. For the normalized antisymmetric coupled two-electron wave functions

$$
\begin{aligned}
& \left|n_{i} n_{j} \lambda_{i} \lambda_{j} u m_{u}\right\rangle_{A} \\
& \quad=T(i, j)\left[\left|n_{i} \lambda_{i} n_{j} \lambda_{j} u m_{u}\right\rangle\right. \\
& \left.\quad-(-1)^{\lambda_{i}+\lambda_{j}-u}\left|n_{j} \lambda_{j} n_{i} \lambda_{i} u m_{u}\right\rangle\right]
\end{aligned}
$$

$(T(i, j)=[1-\delta(i, j)(1-1 / \sqrt{2})] / \sqrt{2}$ is a normalization factor), the submatrix element of $H_{12}^{\text {soo }}$ has the following expression:

$$
\begin{aligned}
& {\left[\lambda_{i} \lambda_{j} u\left\|H_{12}^{\mathrm{soo}}\right\| \lambda_{i^{\prime}} \lambda_{j^{\prime}} d\right]_{A}} \\
& \quad=12(-1)^{\lambda_{i^{\prime}}+\lambda_{j^{\prime}}-d}\left\{T(i, j) T\left(i^{\prime}, j^{\prime}\right)\right\}[u]^{-1 / 2} \\
& \quad \times\left[1-(-1)^{\lambda_{i^{\prime}}+\lambda_{j^{\prime}}-d} P\left(i^{\prime} \leftrightarrow j^{\prime}\right)\right] z_{i j j^{\prime} i^{\prime}}^{\mathrm{soo}}
\end{aligned}
$$

This expression coincides with the formula for the antisymmetric submatrix element given in [7]. Then, taking into account (35) and (43), we obtain

$$
\begin{aligned}
& c z^{\mathrm{soo}} \\
& \quad=\frac{1}{6}(-1)^{l_{i^{\prime}}+l_{j^{\prime}}+L^{\prime}+S^{\prime}+1}\left\{T(i, j) T\left(i^{\prime}, j^{\prime}\right)\right\}^{-1} \\
& \quad \times[L, S]^{1 / 2}\left[\lambda_{i} \lambda_{j} u\left\|H_{12}^{\mathrm{soo}}\right\| \lambda_{i^{\prime}} \lambda_{j^{\prime}} d\right]_{A} .
\end{aligned}
$$

Note that the spin-other-orbit operator has a complex structure described by several terms with different tensorial products (35). In the first approach, $M D_{\alpha \varrho}\left(\gamma, \lambda_{i}, \lambda_{j}, \lambda_{j}^{\prime}, \lambda_{i}^{\prime}, \gamma_{1}, \gamma_{2}, \Lambda_{1}, \Lambda_{2}\right)$ and $G_{\alpha \varrho}^{\left(\Lambda_{1} \Lambda_{2}\right) \underset{m_{\gamma}}{m_{\gamma}}}$ depend on the internal ranks $\gamma_{1}, \gamma_{2}=k, K^{\prime}$ (see also [18]) of each term in $H_{12}^{\text {soo }}$. Differently, in the second approach, only the resulting ranks $\gamma \equiv k \kappa=11$ and $\Gamma=0$ are involved in the determination of the submatrix element of $G_{\alpha \varrho}^{\left(\Lambda_{1} \Lambda_{2}\right) \underset{m_{\gamma}}{(\gamma)}}$ and recoupling coefficients $U E_{\alpha \varrho}\left(\Lambda_{1}, \Lambda_{2}, \gamma, \lambda_{i}, \lambda_{j}, \lambda_{j}^{\prime}, \lambda_{i}^{\prime}, u, d\right)$. All specific information about spin-other-orbit interaction is included only into the factor $c z\left(\gamma, \lambda_{i}, \lambda_{j}, \lambda_{j}^{\prime}, \lambda_{i}^{\prime}, u, d\right)$ which can be found prior to the calculation of the

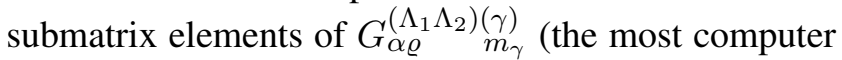
time-consuming part of calculations). Thus, this situation can be more preferable in the case of studying jointly several operators with different tensorial structure.

\section{Summary}

In a second quantization representation two approaches were considered to express an arbitrary twoelectron operator in a coupled tensorial form for multishell atoms. The expressions of both approaches are applicable to the study of the operators representing atomic interactions as well as the opearators describing some effective interactions appearing, for instance, in an atomic MBPT or a coupled cluster method. The first approach is more suitable when one seeks effectively to calculate the matrix elements of (one) particular operator because the internal ranks of operator are 
involved in the calculation of many-electron angular

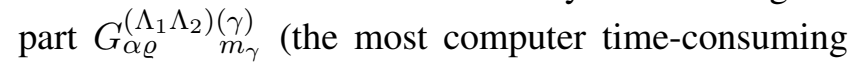
part of calculations). The second approach is superior for the problems where several operators with different tensorial structure are considered, for example, the formation of energy matrix of atomic Hamiltonian in the Breit-Pauli approximation. In this case, only the resulting ranks of operators enter in the expressions for submatrix element of $G_{\alpha \varrho}^{\left(\Lambda_{1} \Lambda_{2}\right)\left(\underset{m}{m_{\gamma}}\right)}$ and the computer codes used for such calculations could be more efficient if they are based on the second approach. The method used in [14] can be explained as the combination of the parts of the first and second approaches. The expressions for $G$ are presented in the mode (i.e. tables and some simple rules to generate the expressions for terms explicitly not given in tables) suitable for easy development of the efficient computer codes which enable to calculate matrix elements for two-electron operator for multishell atoms.

In the case of the consecutive coupling of the resulting moments of shells of many-electron wave function, the matrix element of $G_{\alpha \varrho}^{\left(\Lambda_{1} \Lambda_{2}\right)(\gamma)} m_{\gamma}$ is expressed as simple sums of the products of $6 j$ and / or $9 j$ symbols and matrix elements of operators acting in the space of states formed from a shell of equivalent electrons.

\section{Acknowledgement}

The study was partialy funded by the joint TaiwanBaltic research project.

\section{Appendix}

In the Appendix we present the data necessary to find the expressions for the factors $M D_{\alpha \varrho}, M E_{\alpha \varrho}$, and $U E_{\alpha \varrho}$ of $g g_{\alpha \varrho}^{x}$. In the first column of Table 4, the notation of quantities which enter the expressions for factors $M D_{\alpha \varrho}, M E_{\alpha \varrho}$, and $U E_{\alpha \varrho}$ are given. The second column presents the formulas for the considered quan- tities. To complete the derivation of expressions for the factors $M D_{\alpha \varrho}, M E_{\alpha \varrho}$, and $U E_{\alpha \varrho}$, the formulas

$$
\begin{aligned}
D & \equiv D\left(\lambda_{1}, \lambda_{2}, \gamma_{1}, \gamma_{2}, \gamma, \Lambda_{1}, \Lambda_{2}\right) \\
& =\left[\gamma_{1}, \gamma_{2}, \Lambda_{1}, \Lambda_{2}\right]^{1 / 2}\left\{\begin{array}{lll}
\lambda_{1} & \lambda_{2} & \gamma_{1} \\
\lambda_{1} & \lambda_{2} & \gamma_{2} \\
\Lambda_{1} & \Lambda_{2} & \gamma
\end{array}\right\}, \\
P & \equiv P\left(\lambda_{1}, \lambda_{2}, \gamma_{1}, \gamma_{2}, \gamma, \Lambda_{2}\right) \\
& =\left[\gamma_{1}, \Lambda_{2}\right]^{1 / 2}\left\{\begin{array}{ccc}
\Lambda_{2} & \lambda_{1} & \gamma_{2} \\
\gamma_{1} & \gamma & \lambda_{2}
\end{array}\right\},
\end{aligned}
$$

$$
\begin{aligned}
T N \equiv & T N\left(\lambda_{2}, \lambda_{3}, \gamma_{1}, \gamma_{2}, \gamma, \Lambda_{2}\right)=(-1)^{\gamma+\gamma_{1}+\lambda_{2}+\lambda_{3}} \\
& \times\left[\gamma_{2}, \Lambda_{2}\right]^{1 / 2}\left\{\begin{array}{lll}
\lambda_{3} & \lambda_{2} & \gamma_{2} \\
\gamma_{1} & \gamma & \Lambda_{2}
\end{array}\right\},
\end{aligned}
$$

$$
\begin{aligned}
& E N \equiv E N\left(\lambda_{1}, \lambda_{2}, \lambda_{3}, \gamma_{1}, \gamma_{2}, \gamma, \Lambda_{1}, \Lambda_{2}\right) \\
& =(-1)^{-\gamma_{1}+\lambda_{1}-\lambda_{3}}\left[\gamma_{1}, \gamma_{2}, \Lambda_{1}, \Lambda_{2}\right]^{1 / 2} \\
& \times\left\{\begin{array}{lll}
\lambda_{1} & \lambda_{3} & \gamma_{1} \\
\gamma & \gamma_{2} & \Lambda_{2}
\end{array}\right\}\left\{\begin{array}{ccc}
\lambda_{2} & \lambda_{1} & \gamma_{2} \\
\lambda_{1} & \Lambda_{2} & \Lambda_{1}
\end{array}\right\},
\end{aligned}
$$

$$
P N \equiv P N\left(\lambda_{1}, \lambda_{2}, \lambda_{3}, \gamma_{1}, \gamma_{2}, \gamma, \Lambda_{1}, \Lambda_{2}\right)=(-1)^{1-\Lambda_{2}}
$$

$$
\times\left[\gamma_{1}, \gamma_{2}, \Lambda_{1}, \Lambda_{2}\right]^{1 / 2}\left\{\begin{array}{ccc}
\lambda_{1} & \lambda_{3} & \gamma_{1} \\
\lambda_{2} & \lambda_{3} & \gamma_{2} \\
\Lambda_{1} & \Lambda_{2} & \gamma
\end{array}\right\}
$$

$$
\begin{aligned}
& D V \equiv D V\left(\lambda_{1}, \lambda_{2}, \lambda_{3}, \lambda_{4}, \gamma_{1}, \gamma_{2}, \gamma, \Lambda_{1}, \Lambda_{2}\right) \\
& =(-1)^{-\gamma+\lambda_{3}-\lambda_{4}+\Lambda_{1}}\left[\gamma_{1}, \gamma_{2}, \Lambda_{1}, \Lambda_{2}\right]^{1 / 2} \\
& \times\left\{\begin{array}{ccc}
\lambda_{1} & \lambda_{3} & \gamma_{1} \\
\Lambda_{2} & \lambda_{2} & \Lambda_{1}
\end{array}\right\}\left\{\begin{array}{ccc}
\lambda_{2} & \lambda_{4} & \gamma_{2} \\
\gamma & \gamma_{1} & \Lambda_{2}
\end{array}\right\}
\end{aligned}
$$

have to be used. For example, from Table 2 it follows that the factor $U E_{35}$ of $g g_{35}^{z}$ equals to

$$
D_{32}^{z}=(-1)^{1+\Lambda_{2}} P N\left(\lambda_{1}, \lambda_{2}, \lambda_{3}, u, d, \gamma, \Lambda_{1}, \Lambda_{2}\right),
$$

where $P N$ is defined by (A5).

\section{References}

[1] C. Froese Fischer, Comput. Phys. Commun. 128, 531 (2000). 
[2] C.C. Cannon and A. Derevianko, Phys. Rev. A 69, 030502(R) (2004).

[3] I. Lindgren and M. Morrison, Atomic Many-Body Theory, Springer Series in Chemical Physics, vol. 13, 2nd ed. (Springer-Verlag, Berlin, 1982).

[4] V.A. Dzuba, V.V. Flambaum, and M.G. Kozlov, Phys. Rev. A 54, 3948 (1996).

[5] Z. Rudzikas, Theoretical Atomic Spectroscopy (Cambridge University Press, Cambridge, 1997).

[6] F.A. Parpia, C. Froese Fischer, and I.P. Grant, Comput. Phys. Commun. 175, 745 (2006).

[7] A.P. Jucys and A.J. Savukynas, Mathematical Foundations of the Atomic Theory (Mokslas, Vilnius, 1973) [in Russian].

[8] A. Bar-Shalom, M. Klapisch, and J. Oreg, J. Quant. Spectrosc. Radiat. Transfer 71, 169 (2001).

[9] B.R. Judd, Operator Techniques in Atomic Spectroscopy (McGraw-Hill, New York, 1963).

[10] Z. Rudzikas and J. Kaniauskas, Quasispin and Isospin in the Theory of Atom (Mokslas, Vilnius, 1984) [in Russian].

[11] G. Merkelis, J. Kaniauskas, and Z. Rudzikas, Lietuvos fiz. rink. 25(5), 21 (1985) [in Russian]; Sov. Phys. Collection 25(5), 15 (1985).

[12] G. Merkelis, G. Gaigalas, and Z. Rudzikas, Lietuvos fiz. rink. 25(6), 14 (1985) [in Russian]; Sov. Phys. Collection 25(6), 10 (1985).

[13] P.H.M. Uylings, J. Phys. B 25, 4391 (1992).

[14] G. Gaigalas, Z. Rudzikas, and Ch. Froese Fischer, J. Phys. B 30, 3747 (1996).

[15] G. Merkelis, Phys. Scripta 61, 662 (2000).

[16] W.R. Johnson, Z.W. Liu, and J. Sapirstein, At. Data Nucl. Data Tables 64, 279 (1996).

[17] A.P. Jucys and A.A. Bandzaitis, Theory of Angular Momentum in Quantum Mechanics, 2nd ed. (Mokslas, Vilnius, 1977) [in Russian].

[18] G. Gaigalas, A. Bernotas, Z. Rudzikas, and Ch. Froese Fischer, Phys. Scripta 57, 207 (1998).

\title{
DVIDALELIO OPERATORIAUS SURIŠIMO BŪDAI ATOMINIUOSE SKAIČIAVIMUOSE
}

\author{
R. Juršènas, G. Merkelis \\ Vilniaus universiteto Teorinès fizikos ir astronomijos institutas, Vilnius, Lietuva
}

\begin{abstract}
Santrauka
Aptarta keletas dvielektronio operatoriaus antrinio kvantavimo vaizdavime reiškimo būdų. Vienas iš jų yra labiau priimtinas, kai tiriame vieną kokị nors pasirinktą operatorių. Kitas reiškimo būdas efektyvus, vienu metu generuojant dviejų ir daugiau operatorių,
\end{abstract}

turinčių skirtingas tenzorines savybes, matricas. Pastarasis uždavinys, pavyzdžiui, iškyla formuojant atomo hamiltoniano matricą. Darbe pateiktos išraiškos ịvairiems dvielektronio operatoriaus pavidalams $L S$ ryšio atveju leidžia parengti efektyvius algoritmus konkrečioms problemoms spręsti. 